\title{
The Chain Orientation of Cellulose Flat and Tubular Films Prepared from $\mathrm{N}$-Methylmorpholine $\mathrm{N}$-Oxide Solutions
}

\author{
Yaopeng ZHANG, Huili SHAO, ${ }^{\dagger}$ and Xuechao HU \\ State Key Laboratory for Modification of Chemical Fibers and Polymer Materials, \\ Dong Hua University, Shanghai 200051, People's Republic of China
}

(Received February 15, 2002; Accepted August 2, 2002)

\begin{abstract}
Cellulose flat and tubular films were made from cellulose-aqueous $N$-methylmorpholine $N$-oxide solution system by casting method on nonwovens and blow-extrusion technique, respectively. An attempt was made to disclose the chain orientation of the new cellulose films. It was found that the (11̄0) lattice planes of the flat and tubular films have an uniplanar orientation mode with respect to the film surface. For flat films, the (110) lattice planes are almost unoriented with respect to the machine direction. However, the (110) planes of tubular films have axial orientation with respect to the machine direction. With increasing blow up ratio, both the axial orientation and the crystallinity of tubular films increase. With increasing draw down ratio, only the axial orientation increases while the crystallinity does not change significantly. The three dimensional orientation parameters from polarized Fourier transform infrared spectrometry (FT-IR) spectra by sample tilting indicate that the uniplanar orientation of cellulose chain with respect to the surface of flat film increases when the casting speed increases. Moreover, no axial orientation was found within the plane parallel to the flat film surface. For tubular films, the uniplanar orientation with respect to the film surface increases with the blow up or draw down ratio.

KEY WORDS Cellulose Film / Orientation / $N$-Methylmorpholine $N$-Oxide / Pole Figure / Fourier Transform Infrared Spectrometry (FT-IR)/
\end{abstract}

Recently, $N$-methylmorpholine $N$-oxide (NMMO) as a new organic solvent has been used to dissolve cellulose to prepare novel cellulosic products with improved mechanical properties when compared to materials prepared by the conventional viscose process. ${ }^{1-3}$ Disadvantages of the more than one century old viscose technology are its complicated multi-step processing line and the serious environmental problems arising from the use of $\mathrm{CS}_{2}$. However, the new NMMO process has no chemical reaction and byproducts. It is possible to prepare the cellulose films from NMMO solution using casting method as well as blow-extrusion process in our laboratory. The super-molecular structures of the two types of films must be different due to the different fabricate method. Especially for the blown films, the process of film formation includes two processes simultaneously, that are drawing-down and blowing-up processes. Furthermore, the blowing-up process is a twodimensional elongation process. Therefore, the combination of drawing-down ratio and blow-up ratio may affect the orientation or crystallization of cellulose film.

It is known that X-Ray diffraction is a powerful characterization tool for the elucidation of crystalline microstructure. Nevertheless, it is inaccessible to estimate the degree of orientation in the amorphous phase, which is a very important parameter influencing material properties of polymers. Infrared spectroscopy is a complementary method since vibrational absorptions can arise from chain segments in both amorphous and crystalline phases. In the present paper, X-Ray diffraction techniques are used to reveal and characterize orientation modes in the crystalline regions of the two types of films. In addition, polarized FT-IR spectra for them are also obtained to confirm the changes of orientation for the molecular chains of cellulose.

\section{EXPERIMENTAL}

\section{Film Preparation}

Cellulose sample was purchased from Shanghai Fengxian Pulp Co., China. Fifty percent $N$ methylmorpholine- $N$-oxide (NMMO) water solution obtained from BASF was used as the solvent after concentrating it to $87 \mathrm{wt} \%\left(\mathrm{NMMO} / \mathrm{H}_{2} \mathrm{O}=87 / 13\right.$, expressed as NMMO monohydrate). Moreover, $0.5 \mathrm{wt} \%$ $n$-propylgallate (supplied by Shanghai Reagents Co., China) was added as an antioxidant to avoid oxidation and degradation during the cellulose dissolving process.

The mixing and dissolving of dried cellulose pulp in NMMO monohydrate were carried out at $85^{\circ} \mathrm{C}$ in a container equipped with stirring rake and heating jacket. The cellulose concentration were $9 \mathrm{wt} \%$ and the dope was degassed at $90^{\circ} \mathrm{C}$ for $4 \mathrm{~h}$ before use. ${ }^{4}$

The flat films were obtained by traditional casting method as shown in Figure 1 using the casting ma-

To whom correspondence should be addressed (Tel: +86-21-62373512, Fax: +86-21-62378944, E-mail: hlshao@dhu.edu.cn). 


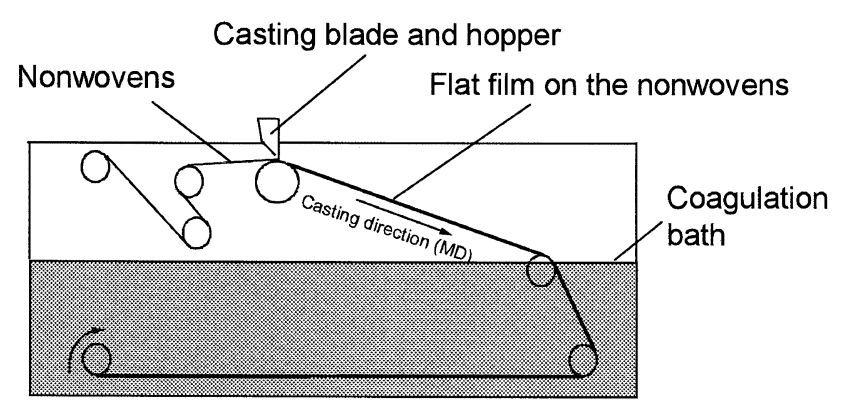

Figure 1. Scheme of the casting process for the preparation of cellulose flat film.

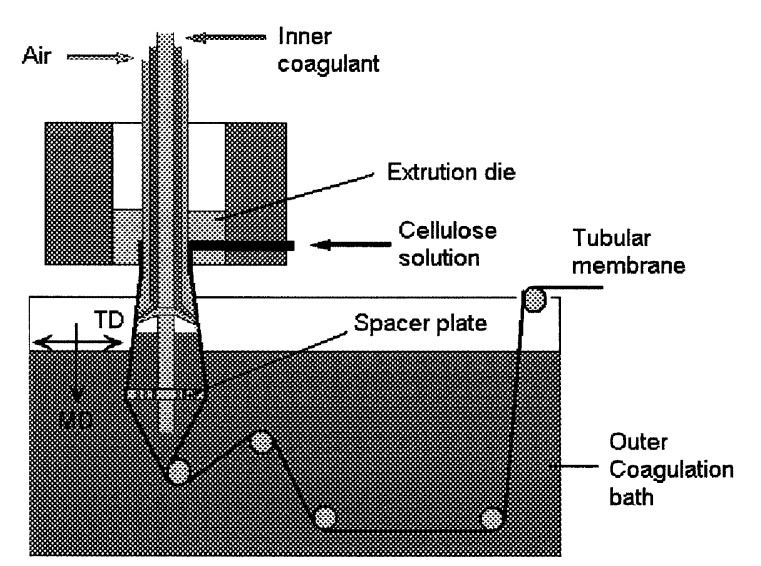

Figure 2. Scheme of the blow extrusion process for the preparation of cellulose tubular film.

chine made by our group. Firstly, the cellulose solution was cast into flat film form on nonwovens fabricated by polyethylene terephthalate (PET) at room temperature. Then the solution with the nonwovens was immersed into pure water bath with the temperature of $25^{\circ} \mathrm{C}$ and wound on a roller.

The tubular films were prepared by the blowextrusion process. The principle of the new route is illustrated in Figure 2. A dope of cellulose with concentration of $9 \mathrm{wt} \%$ in the solvent of NMMO monohydrate was extruded out through a circular blow nozzle with a diameter of up to $30 \mathrm{~mm}$ and temperature of about $90^{\circ} \mathrm{C}$, which shapes the dope in a tube-like fashion. Before coagulated in the coagulation bath, the tube of the highly viscous and elastic dope passed an air-gap about $150 \mathrm{~mm}$. In the air gap, the tube-like shaped dope can be drawn down in machine direction (MD) by adjusting the winding speed and blown up in transverse direction (TD) by controlling the air pressure inside the tube. Both the inner and outer coagulants are pure water with the temperature of $25^{\circ} \mathrm{C}$.

All the films were washed with running water for $24 \mathrm{~h}$ to remove the excess solvent completely before being air-dried with a given dimension for at least $24 \mathrm{~h}$ at ambient temperature.

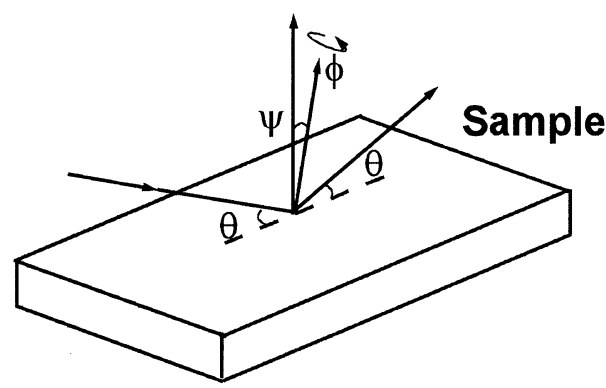

Figure 3. Pole figure measurement.

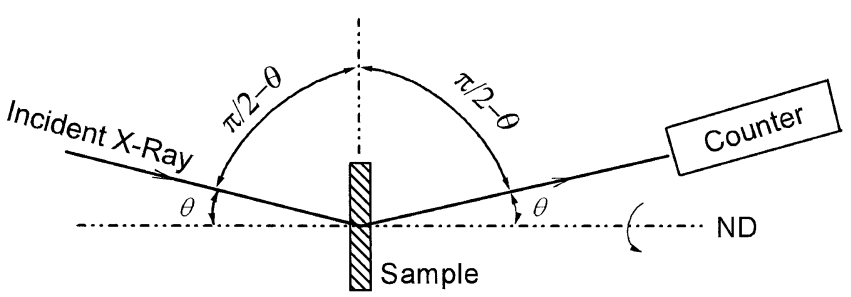

Figure 4. Geometrical arrangement of specimen with respect to X-Ray source and counter for diffractometry in symmetrical transmission method.

\section{X-Ray Diffraction Analysis}

$\mathrm{X}$-Ray diffraction patterns of cellulose films were measured by reflection and transmission methods on an X-Ray diffraction apparatus with scintillation counter (Rigagu D/Max-IIIA). ${ }^{5}$ All experiments were carried out using Nickel filtered $\mathrm{Cu}-K \alpha$ radiation under moderate resolution conditions for $2 \theta$ from 6 to $35^{\circ}$. The crystallinity index was calculated according to the Segal's method. ${ }^{6,7}$

Texture measurements (pole figure) were used to determine the orientation distribution of crystalline grains in polycrystalline cellulose samples. A pole figure is often plotted in polar coordinates consisting of the tilt and rotation angles with respect to a given crystallographic orientation. It is measured at a fixed scattering angle and consists of a series of $\phi$-scans (in-plane rotation around the center of the sample) at different tilt or $\Psi$ (azimuth) angles, as illustrated in Figure 3. The pole figure data are displayed as contour plots or elevation graphs with zero angle in the center. Details of X-Ray texture investigations are given in ref 8 .

A crystalline orientation parameter, $\pi$, of films was estimated from diffraction strength for azimuthal directions by fixing scintillation counter at $2 \theta=20.0^{\circ}$ (corresponding to (110) plane) and by rotating a sample around the normal direction (ND) of film in symmetrical transmission method. The geometrical arrangement of specimen was shown in Figure 4. The $\pi$ was calculated from the following equation: ${ }^{9}$

$$
\pi=\left(180-\Psi_{1 / 2}\right) / 180
$$

where $\Psi_{1 / 2}$ means the half-height width (degree) of 
diffraction intensity. In this paper, cellulose lattice parameter notations were made according to the general crystallographic rule. According to this rule, the $c$-axis coincides with the molecular chain axis and the $b$-axis is larger than the $a$-axis for a monoclinic unit cell. ${ }^{10}$

\section{FT-IR Analysis}

Infrared (IR) spectroscopy is particularly useful for measuring molecular orientation in semicrystalline polymers since IR absorptions frequently exist which are characteristic of amorphous phase material. The degree of chain orientation in the amorphous phase is a very important parameter influencing material properties and is inaccessible to many other characterization techniques such as wide-angle X-Ray scattering. In many polymers, infrared spectra exhibit absorption bands assignable to vibrations of specific chain segments, chemical groups or localized conformations. Each vibrational mode of a molecule has a transition moment vector $M$ associated with it. When infrared radiation is plane-polarized such that the electric vector $E$ is parallel to the transition moment vector, strong absorption occurs; when it is perpendicular, no absorption occurs.

The dichroic ratio, which is used in many references, is a two-dimensional measurement. To completely characterize the orientation of films, three-dimensional measurements are required.

If a highly oriented film sample is sufficiently thin to allow transmission infrared measurements, it has been shown that precise three dimensional orientation information may be obtained by tilting the sample films with respect to the incident infrared beam. ${ }^{11}$ The three principal axes of the film may be defined with $y$ being the machine direction (MD), $x$ the transverse direction (TD), and $z$ the direction perpendicular to the film surface (ND). The axes of the fixed space coordinate are defined with $Y$ being the vertical direction, $X$ the horizontal direction, and $Z$ the direction perpendicular to the $X Y$ plane. An angle $\alpha$ is defined to be the angle of rotation around the $x$-axis. The experimental geometry is shown in Figure 5. The absorbance of bands in three directions $\left(A_{x}, A_{y}, A_{z}\right)$ can be determined by measuring the following four spectral quantities: (a) $A_{x}, y$-axis vertical and incident polarization parallel to the $X$-axis; (b) $A_{y}, y$-axis vertical and incident polarization parallel to the $Y$-axis; (c) $A_{t}^{0}, y$-axis tilted at $\alpha$ from the vertical axis $Y$ and incident polarization parallel to the $X$-axis, (d) $A_{t}^{0}, y$-axis tilted $\alpha$ from the vertical axis $Y$ and incident polarization parallel to the $Y$-axis. $A_{z}$ then can be calculated from the following equation ${ }^{11,12}$

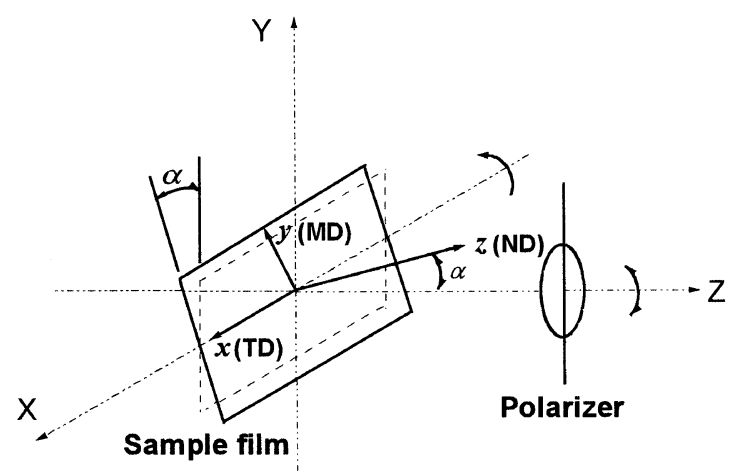

Figure 5. The sample geometry used in the three dimensional orientation measurement.

$$
A_{z}=\frac{A_{t}^{0}\left(1-\sin ^{2} \alpha / n_{z}^{2}\right)^{1 / 2}-A_{y}\left(1-\sin ^{2} \alpha / n_{z}^{2}\right)}{\sin ^{2} \alpha / n_{z}^{2}}
$$

where $n_{z}$ is the index of refraction of the film in the $y z$ plane. Its value may be measured from the following expression $^{11,12}$

$$
n_{z}=\left(\frac{\sin ^{2} \alpha}{1-\left(A_{x} / A_{t}^{90}\right)^{2}}\right)^{1 / 2}
$$

for these cellulose films, a value of 1.4 for $n_{z}$ was determined and a value of $45^{\circ}$ for $\alpha$ was chosen in our experiments.

The classical dichroic ratio in this reference system is $R_{y x}=A_{y} / A_{x}$. Another important factor

$$
A_{0}=\left(A_{x}+A_{y}+A_{z}\right) / 3
$$

represents the absorbance of the band exclusive of contributions due to orientation of the polymer. ${ }^{13}$ This factor is proportional to the amount of chemical structure giving rise to the infrared absorption. The three dimensional orientation parameters: $A_{y} / A_{0}, A_{x} / A_{0}$, and $A_{z} / A_{0}$ can be constructed to determine molecular orientation. For these parameters to have any physical meaning, the direction of the transition moment vector of vibration mode must be known.

The peak band at $1159 \mathrm{~cm}^{-1}$ of the cellulose FT-IR spectra was used to estimate the orientation since it is attributed to anti-symmetrical bridge $\mathrm{C}_{1}-\mathrm{O}-\mathrm{C}_{4}$ stretching band. In particular, the band can be interpreted as representing molecules aligned parallel to the molecular axis and exhibits parallel dichroism. ${ }^{14,15}$

\section{RESULTS AND DISCUSSION}

\section{The Crystalline Orientation of Cellulose Films Ob- tained from X-Ray Diffraction}

It is well known that $c$-axes of cellulose II crystals tend to orient parallel to a drawing direction in $y$-axis 


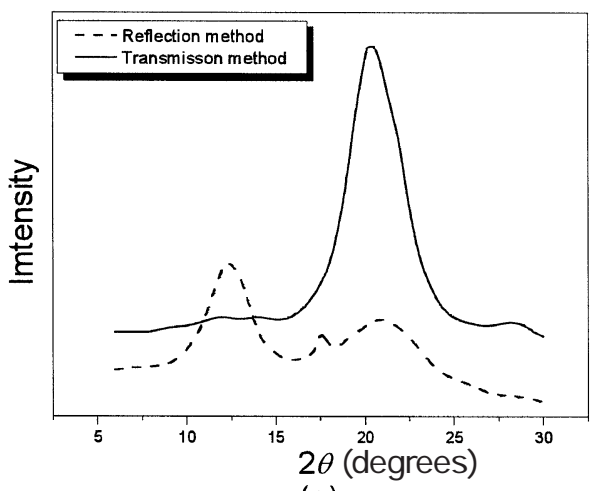

(a)

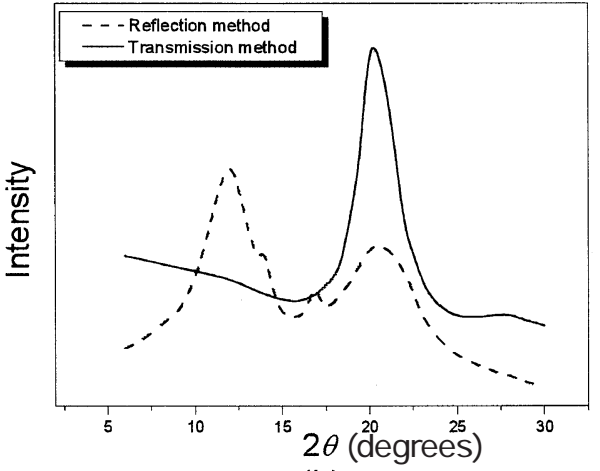

(b)

Figure 6. X-Ray diffraction patterns of cellulose films measured by reflection method and transmission method, (a) flat film, (b) tubular film.

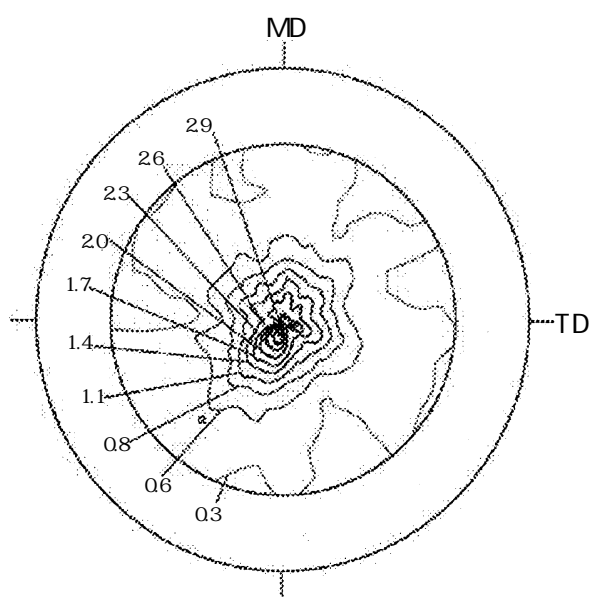

(a)

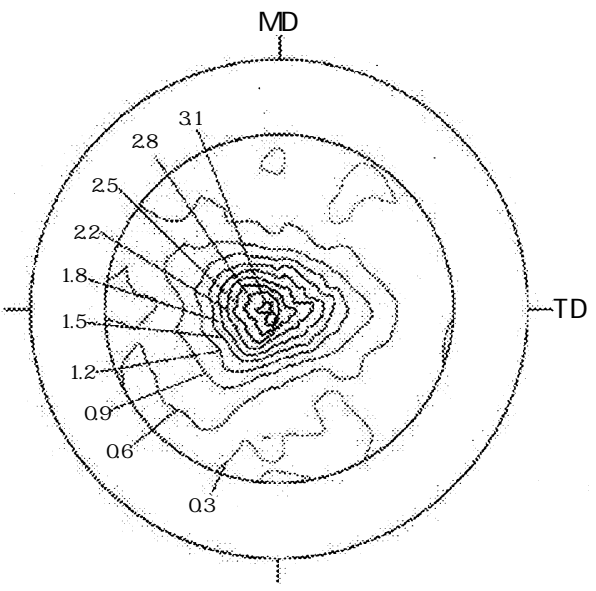

(b)

Figure 7. WAXD pole figures of the (1 $1 \overline{1} 0$ ) lattice planes of cellulose films corresponding to Figure 6. (a) flat film, (b) tubular film, draw down ratio in machine direction larger than transversely.

and $(1 \overline{1} 0)$ planes prefer to orient parallel to the film surface (reference plane). However, in many references ${ }^{16}$ the former orientation (classified as uniaxial orientation) has only been concerned in relation to mechanical properties and processing conditions of regenerated cellulose fibers and films, though the latter orientation (classified as selective uniplanar orientation) could also have important influence on the mechanical properties of cellulose products.

Figure 6 shows the difference of X-Ray diffraction patterns of cellulose films measured using reflection and transmission methods. For the flat and tubular films, the peak intensity corresponding to the

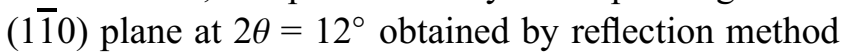
is higher than that obtained by transmission method. However, the peak intensity corresponding to the (110) plane at $2 \theta=20^{\circ}$ obtained by reflection method is less than that obtained by transmission method. Normally, the normal of (110) plane encloses an angle of about $85^{\circ}$ to the normal of the planar-oriented (110) latticeplane. ${ }^{17,18}$ It is also well known that the diffraction peak corresponding to the $(1 \overline{1} 0)$ crystal plane of cellulose exhibits chain orientation parallel to the top surface of the films. ${ }^{19}$ Consequently, the orientation of the (110) plane contributes to the high diffraction peak at $2 \theta=12^{\circ}$ of the intensity curve by reflection method; the orientation of the (110) plane contributes to the high diffraction peak at $2 \theta=20^{\circ}$ of the intensity curve by transmission method.

Mode of orientation of the cellulose films was estimated from X-Ray pole figures of the (110) lattice planes obtained with the texture goniometer in reflection method (Rigaku-Dmax-IIIC). It is recorded with the texture goniometer by moving the sample stepwise around the three axes at a fixed Bragg angle $\left(2 \theta=12^{\circ}\right)$. The sample is characterized by the fixed coordinate system with MD, TD, and ND. The (110) pole figures for selected flat and tubular films are shown in Figure 7. It can be seen from Figure 7 that a very high concentration of poles exists in the immediate neighborhood of the normal to the polymer film. This means that the great majority of the polymer crystallites are oriented with 
their $(1 \overline{1} 0)$ planes nearly parallel to the surface plane of the polymer film. From the sharp peak in the center of the $(1 \overline{1} 0)$ pole figures, it is concluded that there is an uniplanar orientation mode in both the flat and tubular films, which can generally be found in cellulose films. This means that the $(1 \overline{1} 0)$ lattice planes preferably orient towards the macroscopic film surface. For the flat film, a near-circular distribution of the various intensity-levels was achieved (Figure 7a). One of the possible reasons is that the cellulose solution was adhered on the nonwovens and there is no strong stretching in both MD and TD. Therefore, a balanced uniplanar orientation was obtained. However, the pole figure of the blown film has an elliptical intensity distribution which indicating a higher degree of uniplanar orientation in MD than TD (Figure 7b).

In order to investigate whether there is an axial orientation of cellulose chains in the films, the intensity distributions in azimuthal scan of (110) planes were measured in transmission method. The results were shown in Figures 8 and 9.

It is interesting that the intensity of the (110) reflection of the flat films (Figure 8) were very constant as

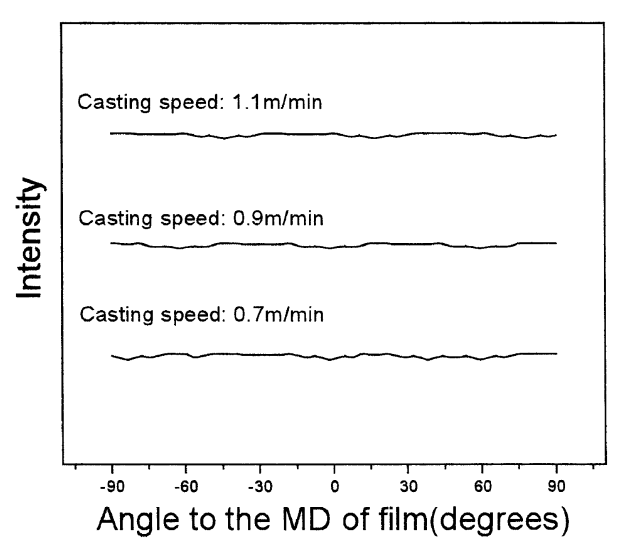

Figure 8. Azimuthal intensity tracings of the (110) arc of the flat films prepared with different casting speed.

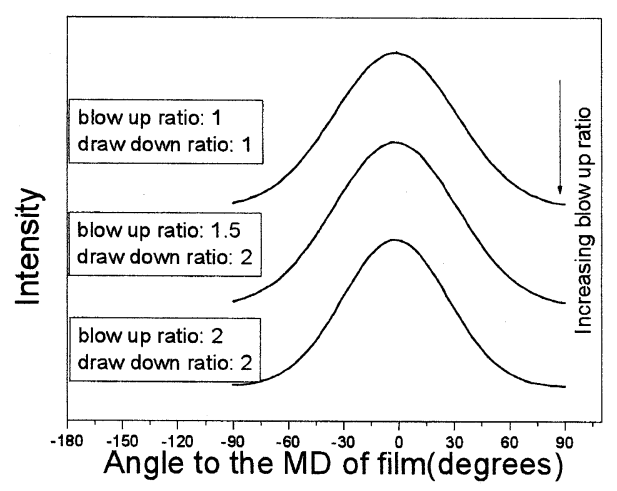

(a) a function of the angle to the MD of film. The casting speed has also no influences on the intensity distributions. That means the (110) plane is almost unoriented to the casting direction (MD). A possible explanation is that the polymer chain was stretched nearly equally in both MD and TD, since cellulose solution is adhered on the nonwovens all through the casting, coagulation and drying procedures. It also should be noticed that the moving speed of the nonwovens is very low in our experiments.

Figure 9 shows the resolution of the (110) azimuthal profiles, which have typical Gaussian distributions, of the tubular films. That means that the (110) planes of the tubular films have preferred axial orientation to MD, which is not observed in the flat films. The orientation parameter, $\pi$, analyzed from WAXD patterns (Figure 9) at (110) planes, and the crystallinity index are shown in Figure 10. The data of Figure 10a show that both the axial orientation and the crystallinity of tubular films increase when the blow up ratio increases. However, the effect of the draw down ratio to the super-molecular structure is somewhat different from that of the blow up ratio. As shown in Figure 10b, the axial orientation increases while the crystallinity does not change significantly even while the draw down ratio increases. That is reasonable because the blowing-up process is a two dimensional elongation process, higher blow up ratio results in greater stretching effect in both MD and TD. Consequently, the normal strain on the cellulose chain within the film surface also increases. Therefore, both higher degrees of axial orientation with respect to MD and uniplanar orientation with respect to the film surface were obtained. The higher degree of the three dimensional order of cellulose chain may contribute to higher crystallinity of the tubular film. However, larger draw down ratio results in greater stretching effect on cellulose chain in MD, which only leads to the predominant increase of the axial orientation with respect to

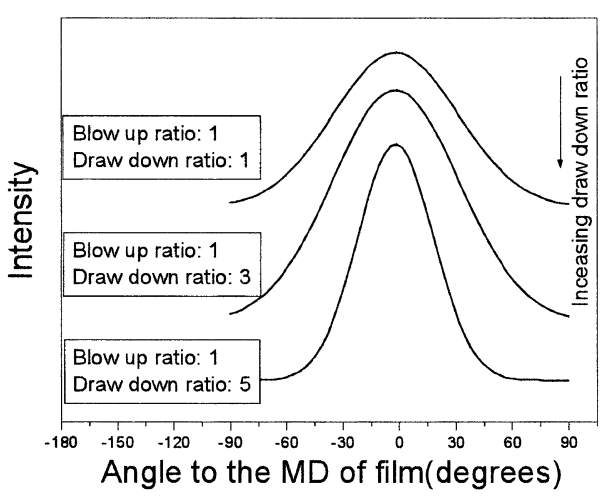

(b)

Figure 9. Azimuthal intensity tracings of the (110) arc of the tubular films prepared with different blow up ratio (a) and draw down ratio (b). 


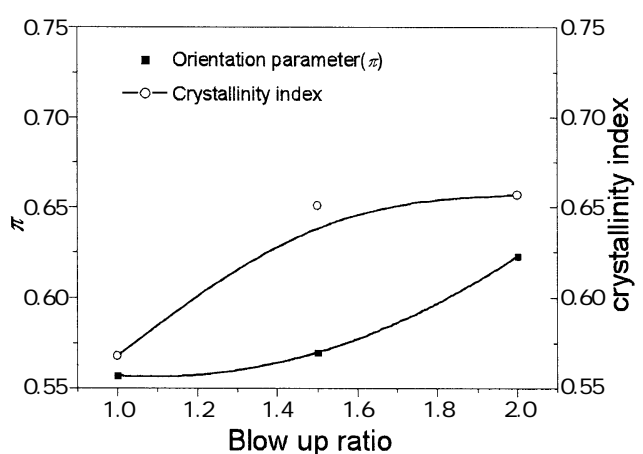

(a)

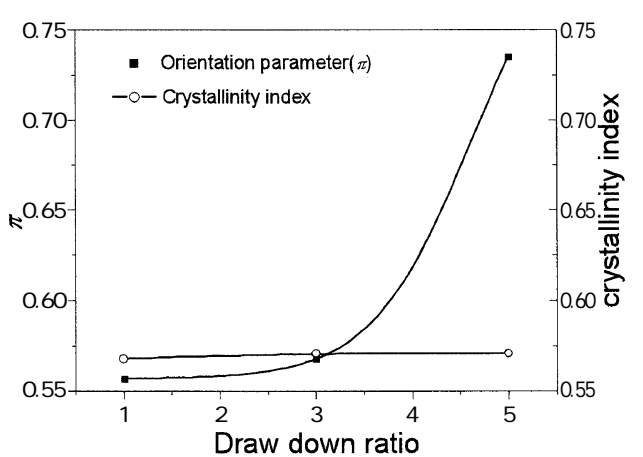

(b)

Figure 10. The effects of blow up ratio (a) and draw down ratio (b) to the orientation parameter and the crystallinity index of the tubular films.

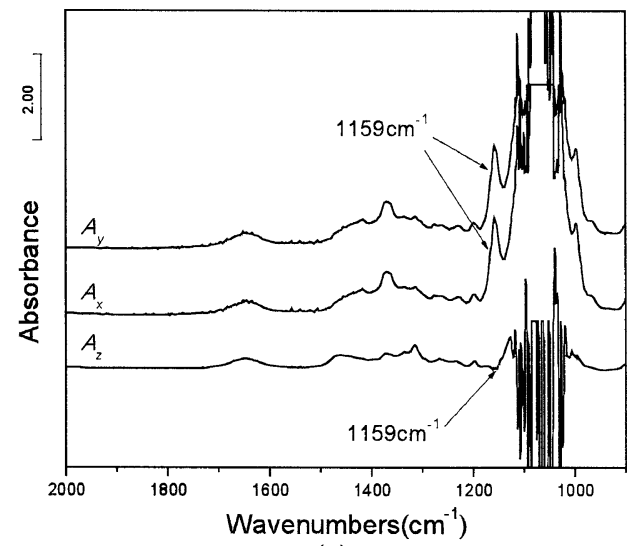

(a)

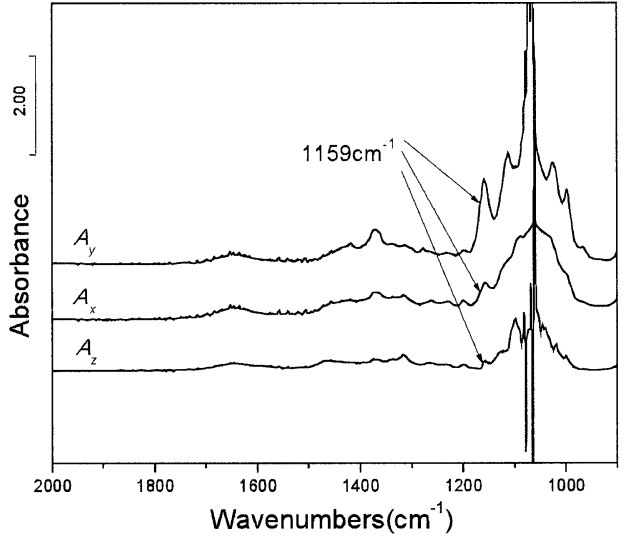

(b)

Figure 11. Determination of $A_{x}, A_{y}$, and $A_{z}$ via sample tilting and spectral subtraction for the cellulose films. (a) flat film, (b) tubular film.

MD. The increase of the two dimensional order of cellulose chain has little contribution to the crystallization.

\section{The Molecular Orientation of Cellulose Films Obtained from FT-IR}

In order to confirm the changes of orientation for the molecular chains of cellulose films, polarized FTIR spectra were obtained using a Nicolet Nexus-670 Fourier transform infrared spectrometer equipped with a polarizer. Operating parameters were chosen such that the wave number accuracy was $\pm 0.02 \mathrm{~cm}^{-1}$ and the spectral resolution was $2 \mathrm{~cm}^{-1}$. The thickness of the films is about $15 \mu \mathrm{m}$. Complete $A_{x}, A_{y}$, and $A_{z}$ can be calculated using the software available on the computer associated with the FT-IR.

The polarized spectra obtained for the two types of cellulose films are shown in Figure 11. For well-assigned parallel vibrations $\left(1159 \mathrm{~cm}^{-1}\right)$ the absorbance measured in $\mathrm{MD}\left(A_{y}\right)$ almost equals to the transverse component $\left(A_{x}\right)$ for flat film (Figure 11a). This suggests that there is no axial orientation in the $x y$ plane of the flat film. However, as shown in Figure 11b, $A_{y}$ value of $1159 \mathrm{~cm}^{-1}$ is much higher than that of $A_{x}$. This implies the molecular chains of tubular film have axial orientation to MD. In addition, the small $A_{z}$ values found in both figures indicate that the molecular chains are perpendicular to the normal of the film plane for both flat and tubular films. In other words, the cellulose chains of these two types of films have uniplanar orientation to the film surface, which coincides with the results from X-Ray diffraction as described before.

From the basic principles of polarized FT-IR analysis focused on $1159 \mathrm{~cm}^{-1}$, it is known that larger value of $A_{y} / A_{x}$ means preferentially orientation to the $y$-axis and lower value of $A_{z} / A_{0}$ means preferentially uniplanar orientation to the $x y$ plane. Figure 12 illustrates the effect of the casting speed on the three dimensional orientation of flat films. That the dichroic ratio of the sample plane $\left(A_{y} / A_{x}\right)$ equals 1 in despite of the increasing casting speed definitely suggests the higher casting speed has no effect on the random orientation within the $x y$ plane. It can also be found that the value of $A_{z} / A_{0}$ decreases while the values of $A_{y} / A_{0}$ and $A_{x} / A_{0}$ increase when the casting speed increases. This means that the degree of uniplanar orientation increases in the case of higher casting speed. This is reasonable because the higher casting speed, the thinner of the film and the larger strain within the film surface plane dur- 
ing the coagulation and drying procedure with a given dimension.

For tubular films, the three dimensional orientation parameters $A_{y} / A_{0}, A_{x} / A_{0}, A_{z} / A_{0}$, and $A_{y} / A_{x}$ are listed in Table I.

By comparing the film of $\mathrm{C} 50 \mathrm{M}$ and $\mathrm{C} 40 \mathrm{M}$, it can be seen that larger blow up ratio results in less $A_{z} / A_{0}$ value and little increase of $A_{y} / A_{x}$ value under the same draw down ratio condition. Consequently, the larger uniplanar orientation of cellulose chain to the $x y$ plane and larger axial orientation to the $y$-axis were obtained when the blow up ratio increases. After the cellulose dope is extruded out from circular nozzle, the polymer chains were stretched in both MD and TD. When the blow up ratio increases, greater stretching effect of the two directions may result in preferential uniplanar orientation of cellulose chains to the film surface. The litthe increase of $A_{y} / A_{x}$ from 1.54952 to 1.57932 and ob-

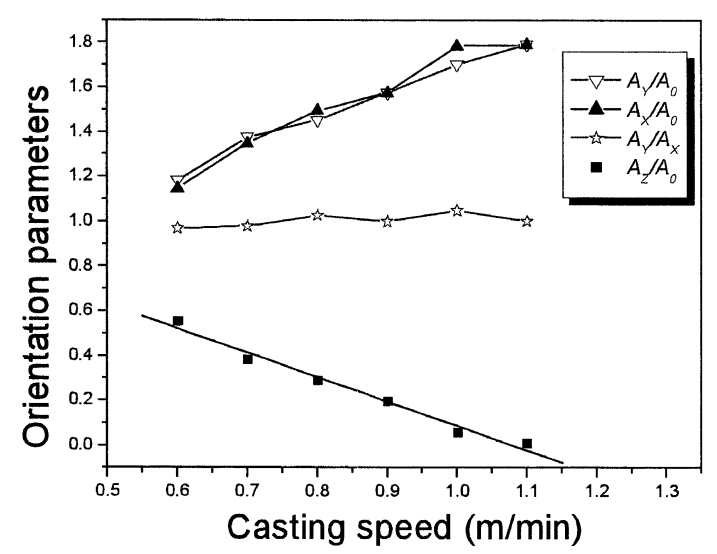

Figure 12. The effect of casting speed on the three dimensional orientation parameters of flat films. vious decrease of $A_{z} / A_{0}$ from 0.42438 to $0.23492 \mathrm{sug}$ gest that the contribution of blow up ratio to the uniplanar orientation is larger than the contribution of blow up ratio to the axial orientation. In addition, it can be found that both $A_{x} / A_{0}$ and $A_{y} / A_{0}$ increase while $A_{z} / A_{0}$ decreases from the comparison of $\mathrm{C} 40 \mathrm{M}$ and $\mathrm{C} 50 \mathrm{M}$. This is because less polymer chains parallel to the ND must result in more polymer chains parallel to the $x y$ plane. Moreover, there are more polymer chains parallel to the $y$-axis than the $x$-axis within this plane.

By comparing the film of $\mathrm{C} 40 \mathrm{M}$ and $\mathrm{C} 40 \mathrm{~F}$, it can be found that larger draw down ratio also results in larger $A_{y} / A_{x}$ value and somewhat less $A_{z} / A_{0}$ value under the same blow up ratio condition. The data indicate that predominant axial and a little higher degree of uniplanar orientation can be obtained in the case of higher draw down ratio. For the film of $\mathrm{C} 25 \mathrm{M}$ that has high value of draw down ratio, the very large $A_{y} / A_{x}$ value suggests that draw down ratio is a dominant factor to influence the axial orientation to the $y$-axis.

From the comparison between the entire column of blow up ratio and $A_{x} / A_{0}$, it can be seen that $A_{x} / A_{0}$ becomes larger with increasing of blow up ratio. Similar result can also be found by comparing the entire column of draw down ratio with $A_{y} / A_{0}$. These two relationships suggest that blowing up contributes to the chain orientation in TD and drawing down contributes to the chain orientation in MD.

\section{CONCLUSION}

The schematic representation for the two modes of orientation for flat and tubular films is shown in Figure 13. There is an uniplanar orientation mode in both

Table I. The three dimensional orientation parameters of tubular films

\begin{tabular}{lccccccc}
\hline Film & $\begin{array}{c}\text { Blow up } \\
\text { ratio }\end{array}$ & $\begin{array}{c}\text { Draw down } \\
\text { ratio }\end{array}$ & $A_{0}$ & $A_{x} / A_{0}$ & $A_{y} / A_{0}$ & $A_{z} / A_{0}$ & $A_{y} / A_{x}$ \\
\hline $\mathrm{C} 50 \mathrm{M}$ & 2 & 2 & 0.91471 & 1.24739 & 1.97002 & 0.23492 & 1.57932 \\
$\mathrm{C} 40 \mathrm{M}$ & 1.5 & 2 & 0.86708 & 1.08295 & 1.67805 & 0.42438 & 1.54952 \\
$\mathrm{C} 40 \mathrm{~F}$ & 1.5 & 3 & 0.66018 & 1.10424 & 1.83282 & 0.35878 & 1.65981 \\
$\mathrm{C} 25 \mathrm{M}$ & 1 & 5 & 0.46413 & 0.52786 & 2.33122 & 0.48853 & 4.41633 \\
\hline
\end{tabular}

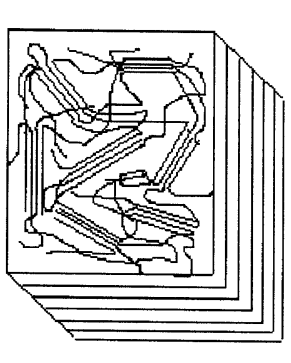

Flat film

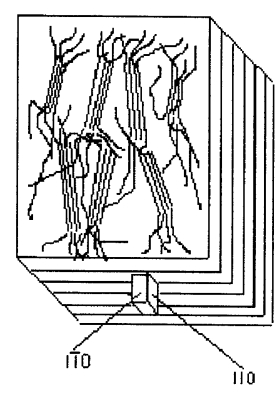

Tubular film

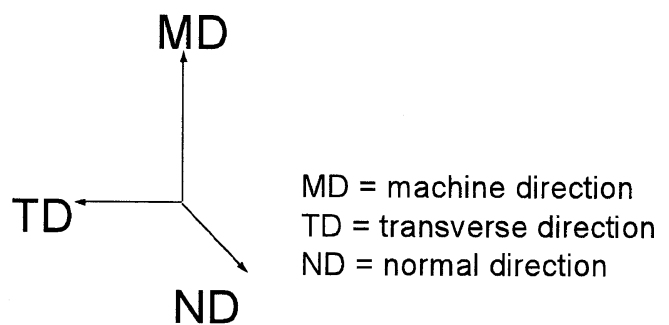

Figure 13. Schematic representation for the two modes of orientation for flat and tubular films. 


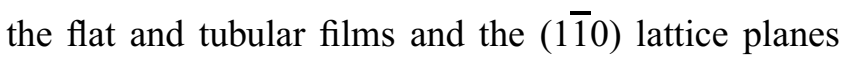
preferentially orientate towards the macroscopic film surface.

For flat films, the (110) plane is almost unoriented with respect to the casting direction (MD). Polymer chain orientation is predominantly in the $x y$ plane. Within this plane very little preferential $x$ or $y$ orientation exists. The uniplanar orientation of cellulose chain to the film surface increases when the casting speed increases.

For tubular films, the (110) planes have preferential axial orientation with respect to the machine direction. With increasing blow up ratio, both the axial orientation and the crystallinity of tubular films increase. With increasing draw down ratio, the axial orientation increases while the crystallinity has no obvious change. The uniplanar orientation of cellulose chain with respect to the surface increases with the blow up or draw down ratio. Blowing up contributes to the molecular orientation in the transverse direction and drawing down contributes to the molecular orientation in the machine direction.

Acknowledgment. The authors would like to thank the Shanghai Priority Discipline Project for the financial support of this project.

\section{REFERENCES}

1. H. Chanzy, A. Peguy, S. Chaunis, and P. Monzie, J. Polym. Sci., Polym. Phys. Ed., 18, 1137 (1980).
2. H. Chanzy, M. Dubéand, and R. H. Marchessault, J. Polym. Sci., Polym. Lett. Ed., 17, 219 (1979).

3. H. Burger, R. Maron, C. Michels, and K. Mieck, Fibre Chemistry, 28, 22 (1996).

4. Y. P. Zhang, H. L. Shao, C. X. Wu, and X. C. Hu, Macromol. Biosci., 1, 141 (2001).

5. T. Takahashi, Sen'i Gakkaishi, 25, 80 (1969).

6. L. Segal, J. J. Creely, A. E. Martin Jr., and C. M. Conrad, Text. Res. J., 29, 786 (1959).

7. A. Isogai and M. Usuda, Sen'i Gakkaishi, 46, 324 (1990).

8. L. E. Alexander, "X-Ray Diffraction Methods in Polymer Science”, John Wiley \& Sons, Inc., New York, N.Y., 1969, p 198.

9. C. Yamane, M. Mori, M. Saito, and K. Okajima, Polym. J., 28, 1039 (1996).

10. T. Kondo in "Polysaccharides: Structural Diversity and Functional Versatility of Polysaccharides", S. Dumitriu, Ed., Marcel Dekker, New York, N.Y., 1998, chapt. 4, pp 131-172.

11. D. Y. Shen, S. E. Molis, and S. L. Hsu, Polym. Eng. Sci., 23, 543 (1983).

12. A. Garton, D. J. Carlsson, and D. M. Wiles, Appl. Spectrosc., 35, 432 (1981).

13. J. L. Koenig, S. W. Cornell, and D. E. Witenhafer, J. Polym. Sci., Part A: Polym. Chem., 5, 301 (1967).

14. R. H. Marchessault and C. Y. Liang, J. Polym. Sci., 43, 71 (1960).

15. Y. Kataoka and T. Kondo, Macromolecules, 31, 760 (1998).

16. E. Togawa and T. Kondo, J. Polym. Sci., Polym. Phys. Ed., 37, 451 (1999).

17. K. J. Kolpak, M. Weih, and J. Blackwell, Polymer, 19, 123 (1978).

18. H.-P. Fink, P. Weigel, H. J. Purz, and A. Bohn, Recent Research Development in Polymer Science, 2, 387 (1998).

19. G. Yang, L. Zhang, and H. Feng, J. Membr. Sci., 161, 31 (1999). 